International Journal of Pure and Applied Mathematics

Volume 90 No. $3 \quad 2014$, 297-308

ISSN: 1311-8080 (printed version); ISSN: 1314-3395 (on-line version)

url: http://www.ijpam.eu

doi: http://dx.doi.org/10.12732/ijpam.v90i3.4

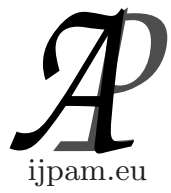

\title{
V-TRANSFORMATION OF STRONG VARIETIES \\ OF PARTIAL ALGEBRAS
}

\author{
Saofee Busaman \\ Department of Mathematics and Computer Science \\ Faculty of Science and Technology \\ Prince of Songkla University \\ Pattani Campus \\ 181 Rusamilae Muang Pattani 94000, THAILAND
}

\begin{abstract}
In this paper, we define $\mathrm{V}$-transformation and prove that V- transformation is a bijective if $\mathrm{V}$ - transformation is a composition of binary relations with inverse of itself then equal relation delte of C-terms.
\end{abstract}

AMS Subject Classification: 08A55, 08B15, 08B25

Key Words: partial algebra, strong variety, tree transformation, $V$-transformation

\section{Introduction}

Let $P^{n}(A):=\left\{f: A^{n} \multimap \rightarrow A\right\}$ be the set of all $n$-ary partial operations defined on the set $A$ and let $P(A):=\bigcup_{n=1}^{\infty} P^{n}(A)$ be the set of all partial operations on A. A partial algebra $\mathcal{A}=\left(A ;\left(f_{i}^{A}\right)_{i \in I}\right)$ of type $\tau=\left(n_{i}\right)_{i \in I}$ is a pair consisting of a set $A$ and an indexed set $\left(f_{i}^{A}\right)_{i \in I}$ of partial operations where $f_{i}^{A}$ is $n_{i}$-ary.

Received: June 29, 2013

(c) 2014 Academic Publications, Ltd. url: www.acadpubl.eu 
Let $P A l g(\tau)$ be the class of all partial algebras of type $\tau$. Let $W_{\tau}\left(X_{n}\right)$ be the set of all $n$-ary terms of type $\tau$ and let $W_{\tau}\left(X_{n}\right)^{\mathcal{A}}$ be the set of all $n$-ary term operations induced by $n$-ary terms on the partial algebra $\mathcal{A}$. For the definition of a term operation $t^{\mathcal{A}}$ induced by the term $t$ on the partial algebra $\mathcal{A}$ (see [1]). Different from the total case, the set $W_{\tau}\left(X_{n}\right)^{\mathcal{A}}$ is in general a proper subset of $P^{n}(A)$. Therefore in [1] F. Börner introduced another concept of terms for partial algebras.

Let $X$ be an alphabet and let $\left\{f_{i} \mid i \in I\right\}$ be a set of operation symbols of type $\tau$, where each $f_{i}$ has arity $n_{i}$ and $X \cap\left\{f_{i} \mid i \in I\right\}=\phi$. We will need additional symbols $\varepsilon_{j}^{k} \notin X$, for every $k \in \mathbb{N}^{+}:=\mathbb{N} \backslash\{0\}$ and $1 \leq j \leq k$. Let $X_{n}=\left\{x_{1}, \ldots, x_{n}\right\}$ be an $n$-element alphabet. The set of all $n$-ary terms of type $\tau$ over $X_{n}$ is defined inductively as follows (see [1]):

(i) every $x_{i} \in X_{n}$ is an $n$-ary term of type $\tau$;

(ii) if $w_{1}, \ldots, w_{k}$ are $n$-ary terms of type $\tau$, then $\varepsilon_{j}^{k}\left(w_{1}, \ldots, w_{k}\right)$ is an $n$-ary term of type $\tau$ for all $1 \leq j \leq k$ and all $k \in \mathbb{N}^{+}$;

(iii) if $w_{1}, \ldots, w_{n}$ are $n$-ary terms of type $\tau$ and if $f_{i}$ is an $n_{i}$-ary operation symbol, then $f_{i}\left(w_{1}, \ldots, w_{n_{i}}\right)$ is an $n$-ary term of type $\tau$.

Let $W_{\tau}^{C}\left(X_{n}\right)$ be the set of all $n$-ary terms of type $\tau$. Then $W_{\tau}^{C}(X):=$ $\bigcup_{n=1}^{\infty} W_{\tau}^{C}\left(X_{n}\right)$ denotes the set of all terms of this type.

On the sets $W_{\tau}^{C}\left(X_{n}\right)$ we may introduce the following superposition operations. Let $w_{1}, \ldots, w_{m}$ be $n$-ary terms and let $t$ be an $m$-ary term. Then we define an $n$-ary term $\bar{S}_{n}^{m}\left(t, w_{1}, \ldots, w_{m}\right)$ inductively by the following steps:

(i) For $t=x_{j}, 1 \leq j \leq m$ (m-ary variable $)$, we define $\bar{S}_{n}^{m}\left(x_{j}, w_{1}, \ldots, w_{m}\right)=$ $w_{j}$.

(ii) For $t=\varepsilon_{j}^{k}\left(s_{1}, \ldots, s_{k}\right)$ we set

$$
\bar{S}_{n}^{m}\left(t, w_{1}, \ldots, w_{m}\right)=\varepsilon_{j}^{k}\left(\bar{S}_{n}^{m}\left(s_{1}, w_{1}, \ldots, w_{m}\right), \ldots, \bar{S}_{n}^{m}\left(s_{k}, w_{1}, \ldots, w_{m}\right)\right),
$$

where $s_{1}, \ldots, s_{k}$ are $m$-ary, for all $k \in \mathbb{N}^{+}$and $1 \leq j \leq k$.

(iii) For $t=f_{i}\left(s_{1}, \ldots, s_{n_{i}}\right)$ we set

$$
\bar{S}_{n}^{m}\left(t, w_{1}, \ldots, w_{m}\right)=f_{i}\left(\bar{S}_{n}^{m}\left(s_{1}, w_{1}, \ldots, w_{m}\right), \ldots, \bar{S}_{n}^{m}\left(s_{n_{i}}, w_{1}, \ldots, w_{m}\right)\right),
$$

where $s_{1}, \ldots, s_{n_{i}}$ are $m$-ary. 
This defines an operation

$$
\bar{S}_{n}^{m}: W_{\tau}^{C}\left(X_{m}\right) \times\left(W_{\tau}^{C}\left(X_{n}\right)\right)^{m} \longrightarrow W_{\tau}^{C}\left(X_{n}\right),
$$

which describes the superposition of terms.

The term clone of type $\tau$ is the heterogeneous algebra

$$
\text { clone } \tau^{C}:=\left(\left(W_{\tau}^{C}\left(X_{n}\right)\right) ; \bar{S}_{n}^{m}, e_{j}^{k}\right)_{n, m, k \in \mathbb{N}^{+}, 1 \leq j \leq k},
$$

where $e_{j}^{k}:=x_{j} \in X_{k}, 1 \leq j \leq k$.

Every $n$-ary term $w \in W_{\tau}^{C}\left(X_{n}\right)$ induces an $n$-ary term operation $w^{\mathcal{A}}$ of any partial algebra $\mathcal{A}=\left(A ;\left(f_{i}^{\mathcal{A}}\right)_{i \in I}\right)$ of type $\tau$. For $a_{1}, \ldots, a_{n} \in A$, the value $w^{\mathcal{A}}\left(a_{1}, \ldots, a_{n}\right)$ is defined in the following inductive way (see [1]) :

(i) If $w=x_{i}$ then $w^{\mathcal{A}}=x_{i}^{\mathcal{A}}=e_{i}^{n, A}$, where $e_{i}^{n, A}$ is as usual the $n$-ary total projection on the $i$-th component.

(ii) If $w=\varepsilon_{j}^{k}\left(w_{1}, \ldots, w_{k}\right)$ and we assume that $w_{1}^{\mathcal{A}}, \ldots, w_{k}^{\mathcal{A}}$ are the term operations induced by the terms $w_{1}, \ldots, w_{k}$ and that the $w_{i}^{\mathcal{A}}\left(a_{1}, \ldots, a_{n}\right)$ are defined for $1 \leq i \leq k$, then $w^{\mathcal{A}}\left(a_{1}, \ldots, a_{n}\right)$ is defined and $w^{\mathcal{A}}\left(a_{1}, \ldots, a_{n}\right)=$ $w_{j}^{\mathcal{A}}\left(a_{1}, \ldots, a_{n}\right)$.

(iii) Now assume that $w=f_{i}\left(w_{1}, \ldots, w_{n_{i}}\right)$ where $f_{i}$ is an $n_{i}$-ary operation symbol, and assume that the $w_{j}^{\mathcal{A}}\left(a_{1}, \ldots, a_{n}\right)$ are defined, with values $w_{j}^{\mathcal{A}}\left(a_{1}, \ldots, a_{n}\right)=b_{j}$ for $1 \leq j \leq n_{i}$. If $f_{i}^{A}\left(b_{1}, \ldots, b_{n_{i}}\right)$ is defined, then $w^{\mathcal{A}}\left(a_{1}, \ldots, a_{n}\right)$ is defined and

$$
w^{\mathcal{A}}\left(a_{1}, \ldots, a_{n}\right)=f_{i}^{A}\left(w_{1}^{\mathcal{A}}\left(a_{1}, \ldots, a_{n}\right), \ldots, w_{n_{i}}^{\mathcal{A}}\left(a_{1}, \ldots, a_{n}\right)\right) .
$$

Definition 1. (see [4]) A pair $t_{1} \approx t_{2} \in W_{\tau}^{C}(X)^{2}$ is called a strong identity in a partial algebra $\mathcal{A}$ (in symbols $\mathcal{A} \models_{s} t_{1} \approx t_{2}$ ) iff $t_{1}^{\mathcal{A}}$ is defined whenever $t_{2}^{\mathcal{A}}$ is defined and conversely and $t_{1}^{\mathcal{A}}=t_{2}^{\mathcal{A}}$ on the common domain, i.e. the induced partial term operations $t_{1}^{\mathcal{A}}$ and $t_{2}^{\mathcal{A}}$ are equal.

Let $K \subseteq P A l g(\tau)$ be a class of partial algebras of type $\tau$ and $\Sigma \subseteq W_{\tau}^{C}(X)^{2}$. Consider the connection between $\operatorname{PAlg}(\tau)$ and $W_{\tau}^{C}(X)^{2}$ given by the following two operators:

$$
I d^{s}: \mathcal{P}(P A l g(\tau)) \rightarrow \mathcal{P}\left(W_{\tau}^{C}(X)^{2}\right)
$$

and

$$
\operatorname{Mod}^{s}: \mathcal{P}\left(W_{\tau}^{C}(X)^{2}\right) \rightarrow \mathcal{P}(P A l g(\tau))
$$


with

$$
\begin{aligned}
I d^{s} K & :=\left\{s \approx t \in W_{\tau}^{C}(X)^{2} \mid \forall \mathcal{A} \in K\left(\mathcal{A} \models_{s} s \approx t\right)\right\} \quad \text { and } \\
\operatorname{Mod}^{s} \Sigma & :=\left\{\mathcal{A} \in P \operatorname{PAlg}(\tau) \mid \forall s \approx t \in \Sigma\left(\mathcal{A} \models_{s}^{\models} s \approx t\right)\right\} .
\end{aligned}
$$

Clearly, the pair $\left(\operatorname{Mod}^{s}, I d^{s}\right)$ is a Galois connection between $P \operatorname{Alg}(\tau)$ and $W_{\tau}^{C}(X)^{2}$. We have two closure operators $M o d^{s} I d^{s}$ and $I d^{s} M o d^{s}$ and their sets of fixed points.

Definition 2. Let $V \subseteq P \operatorname{Alg}(\tau)$ be a class of partial algebras of type $\tau$. The class $V$ is called a strong variety of partial algebras if $V=\operatorname{Mod}^{s} I d^{s} V$.

Definition 3. (see [5]) Let $\left\{f_{i} \mid i \in I\right\}$ be a set of operation symbols of type $\tau$ and $W_{\tau}^{C}(X)$ be the set of all terms of this type. A mapping $\sigma:\left\{f_{i} \mid i \in\right.$ $I\} \longrightarrow W_{\tau}^{C}(X)$ which maps each $n_{i}$-ary fundamental operation $f_{i}$ to a term of arity $n_{i}$ is called a hypersubstitution of type $\tau$.

Any hypersubstitution $\sigma$ of type $\tau$ can be extended to map $\widehat{\sigma}: W_{\tau}^{C}(X) \longrightarrow$ $W_{\tau}^{C}(X)$ defined for all terms, in the following way:

(i) $\widehat{\sigma}\left[x_{i}\right]=x_{i}$ for every $x_{i} \in X_{n}$,

(ii) $\widehat{\sigma}\left[\varepsilon_{j}^{k}\left(s_{1}, \ldots, s_{k}\right)\right]=\bar{S}_{n}^{k}\left(\varepsilon_{j}^{k}\left(x_{1}, \ldots, x_{k}\right), \widehat{\sigma}\left[s_{1}\right], \ldots, \widehat{\sigma}\left[s_{k}\right]\right)$, where $s_{1}, \ldots, s_{k} \in$ $W_{\tau}^{C}\left(X_{n}\right)$,

(iii) $\widehat{\sigma}\left[f_{i}\left(t_{1}, \ldots, t_{n_{i}}\right)\right]=\bar{S}_{n}^{n_{i}}\left(\sigma\left(f_{i}\right), \widehat{\sigma}\left[t_{1}\right], \ldots, \widehat{\sigma}\left[t_{n_{i}}\right]\right)$, where $t_{1}, \ldots, t_{n_{i}} \in W_{\tau}^{C}\left(X_{n}\right)$.

Let $\operatorname{Var}(t)$ be the set of all variables occurring in the term $t$.

Definition 4. (see [3]) The hypersubstitution $\sigma$ is called regular if

$$
\operatorname{Var}\left(\sigma\left(f_{i}\right)\right)=\left\{x_{1}, \ldots, x_{n_{i}}\right\}, \quad i \in I .
$$

Let $\operatorname{Hyp}_{R}^{C}(\tau)$ be the set of all regular hypersubstitutions of type $\tau$.

Lemma 5. (see [5]) Let $\sigma_{1}, \sigma_{2} \in \operatorname{Hyp}_{R}^{C}(\tau)$. Then $\left(\widehat{\sigma}_{1} \circ \sigma_{2}\right)^{\wedge}=\widehat{\sigma}_{1} \circ \widehat{\sigma}_{2}$, where $\circ$ is the usual composition of functions.

Now we define a product of regular hypersubstitutions is the usual way, by $\sigma_{1} \circ_{h} \sigma_{2}:=\widehat{\sigma}_{1} \circ \sigma_{2}$.

Theorem 6. (see [5]) The algebra $\left(\operatorname{Hyp}_{R}^{C}(\tau) ; \circ_{h}, \sigma_{i d}\right)$ with $\sigma_{i d}\left(f_{i}\right)=f_{i}\left(x_{1}\right.$, $\left.\ldots, x_{n_{i}}\right)$ is a monoid. let

Let $\mathcal{A}=\left(A ;\left(f_{i}^{A}\right)_{i \in I}\right)$ be a partial algebra of type $\tau$ and $\sigma \in H y p_{R}(\tau)$. We

$$
\sigma(\mathcal{A}):=\left(A ;\left(\sigma\left(f_{i}\right)^{\mathcal{A}}\right)_{i \in I}\right)
$$


which is called derived algebra of type $\tau$, where $\sigma\left(f_{i}\right)^{\mathcal{A}}$ is the term operation induced by the term $\sigma\left(f_{i}\right)$ on the algebra $\mathcal{A}$.

\section{Tree Transformations}

Definition 7. Let $\sigma$ be a regular hypersubstitution. Then

$$
T_{\sigma}:=\left\{(t, \widehat{\sigma}[t]) \mid t \in W_{\tau}^{C}(X)\right\}
$$

is called the tree transformation defined by the regular hypersubstitution $\sigma$.

We denote by $T_{\sigma_{1}} \circ T_{\sigma_{2}}$ the composition of the tree transformations $T_{\sigma_{1}}$ and $T_{\sigma_{2}}$. Since a tree transformation $T_{\sigma}$ is a relation, we can consider inverses, domains and ranges of such transformations under the relational composition ○. We define

$$
T_{H y p_{R}^{C}(\tau)}:=\left\{T_{\sigma} \mid \sigma \in \operatorname{Hyp}_{R}^{C}(\tau)\right\}
$$

and prove that

Theorem 8. $\left(T_{H y p_{R}^{C}(\tau)} ; \circ, T_{\sigma_{i d}}\right)$ is a monoid which is isomorphic to the monoid $\operatorname{Hyp}_{R}^{C}(\tau)$ of all regular hypersubstitutions of type $\tau$.

Proof. We define a mapping $\varphi: H y p_{R}^{C}(\tau) \rightarrow T_{H y p_{R}^{C}(\tau)}$ by $\sigma \mapsto T_{\sigma}$. Clearly, $\varphi$ is well-defined and surjective.

(i): We show that $T_{\sigma_{1}} \circ T_{\sigma_{2}}=T_{\sigma_{1} \circ_{h} \sigma_{2}}$, i.e. $\varphi\left(\sigma_{1} \circ_{h} \sigma_{2}\right)=\varphi\left(\sigma_{1}\right) \circ \varphi\left(\sigma_{2}\right)$. Indeed, we have

$$
\begin{aligned}
\left(t, t^{\prime \prime}\right) \in T_{\sigma_{1}} \circ T_{\sigma_{2}} & \Leftrightarrow \exists t^{\prime}\left(\left(t, t^{\prime}\right) \in T_{\sigma_{2}} \text { and }\left(t^{\prime}, t^{\prime \prime}\right) \in T_{\sigma_{1}}\right) \\
& \Leftrightarrow t^{\prime}=\widehat{\sigma}_{2}[t] \text { and } t^{\prime \prime}=\widehat{\sigma}_{1}\left[t^{\prime}\right] \\
& \Leftrightarrow t^{\prime \prime}=\widehat{\sigma}_{1}\left[\widehat{\sigma}_{2}[t]\right] \\
& \Leftrightarrow t^{\prime \prime}=\left(\sigma_{1} \circ_{h} \sigma_{2}\right)^{\wedge}[t] \\
& \Leftrightarrow\left(t, t^{\prime \prime}\right) \in T_{\sigma_{1} \circ_{h} \sigma_{2} .}
\end{aligned}
$$

This shows that $T_{H y p_{R}^{C}(\tau)}$ is closed under composition and that $\varphi$ preserves the operation.

(ii): We show that $\varphi$ is one-to-one.

Assume that $T_{\sigma_{1}}=T_{\sigma_{2}}$. Then for all $t \in W_{\tau}^{C}(X)$ we have $\widehat{\sigma}_{1}[t]=\widehat{\sigma}_{2}[t]$. But this means that for all operation symbols $f_{i}$ we also have

$$
\widehat{\sigma}_{1}\left[f_{i}\left(x_{1}, \ldots, x_{n_{i}}\right)\right]=\sigma_{1}\left(f_{i}\right)=\sigma_{2}\left(f_{i}\right)=\widehat{\sigma}_{2}\left[f_{i}\left(x_{1}, \ldots, x_{n_{i}}\right)\right]
$$

and, therefore, $\sigma_{1}=\sigma_{2}$.

Finally, since $T_{\sigma_{1}} \circ T_{\sigma_{2}}=T_{\sigma_{1} \circ_{h} \sigma_{2}}$, the tree transformation $T_{\sigma_{i d}}$ is an identity element with respect to the composition $\circ$. 
Theorem 8 allows us to describe properties of the relation $T_{\sigma}$ by properties of the regular hypersubstitution $\sigma$ and conversely.

Theorem 9. Let $\sigma \in H y p_{\tau}^{C}(X)$ be a regular hypersubstitution of type $\tau$ and let $T_{\sigma}$ be the corresponding tree transformation. Then

(i) $T_{\sigma}$ is transitive iff $\sigma$ is idempotent,

(ii) $T_{\sigma}$ is reflexive iff $\sigma=\sigma_{i d}$,

(iii) $T_{\sigma}$ is symmetric iff $\sigma \circ_{h} \sigma=\sigma_{i d}$.

Proof. (i): When $\sigma$ is idempotent, we have $T_{\sigma \circ_{h} \sigma}=T_{\sigma} \circ T_{\sigma}=T_{\sigma}$ by Theorem 8, and $T_{\sigma}$ is transitive. Conversely, when $T_{\sigma}$ is transitive, we have $T_{\sigma} \circ T_{\sigma} \subseteq T_{\sigma}$, so that $T_{\sigma \circ{ } \sigma} \subseteq T_{\sigma}$. Then

$$
\left(t,\left(\sigma \circ_{h} \sigma\right)^{\wedge}[t]\right) \in T_{\sigma \circ_{h} \sigma} \Rightarrow\left(t,\left(\sigma \circ_{h} \sigma\right)^{\wedge}[t]\right) \in T_{\sigma} \Rightarrow\left(\sigma \circ_{h} \sigma\right)^{\wedge}[t]=\widehat{\sigma}[t],
$$

for all $t \in W_{\tau}^{C}(X)$, and $\sigma$ is idempotent.

(ii): Assume that $T_{\sigma}$ is reflexive, so that $T_{\sigma_{i d}}=\Delta_{W_{\tau}^{C}(X)} \subseteq T_{\sigma}$. Therefore $(t, t) \in T_{\sigma}$ for all $t \in W_{\tau}^{C}(X)$ and then $\widehat{\sigma}[t]=t$ for all $t \in W_{\tau}^{C}(X)$, making $\sigma=\sigma_{i d}$.

If conversely $\sigma=\sigma_{i d}$, then $T_{\sigma_{i d}}=\left\{\left(t, \widehat{\sigma}_{i d}[t]\right) \mid t \in W_{\tau}^{C}(X)\right\}=\{(t, t) \mid t \in$ $\left.W_{\tau}^{C}(X)\right\}=\Delta_{W_{\tau}^{C}(X)}$ and $T_{\sigma}$ is reflexive.

(iii): If $T_{\sigma}$ is symmetric, then for all $t \in W_{\tau}^{C}(X)$ we have

$$
(t, \widehat{\sigma}[t]) \in T_{\sigma} \Rightarrow(\widehat{\sigma}[t], t) \in T_{\sigma} .
$$

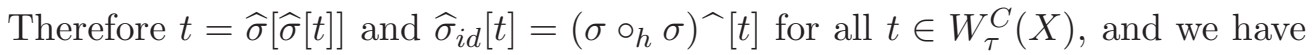
$\sigma \circ_{h} \sigma=\sigma_{i d}$.

If conversely $\sigma \circ_{h} \sigma=\sigma_{i d}$ then we have $T_{\sigma \circ_{h} \sigma}=T_{\sigma} \circ T_{\sigma}=T_{\sigma_{i d}}$. But this means $T_{\sigma}=\left(T_{\sigma}\right)^{-1}$, and $T_{\sigma}$ is symmetric.

A tree transformation is called injective if $\sigma$ is injective, i.e., if from $\widehat{\sigma}[t]=$ $\widehat{\sigma}\left[t^{\prime}\right]$ follows $t=t^{\prime}$, and $T_{\sigma}$ is called surjective if $\sigma$ is surjective.

In general, the range of a tree transformation $\sigma$, i.e. the set

$$
\widehat{\sigma}\left(W_{\tau}^{C}(X)\right)=\left\{t^{\prime} \mid \exists t \in W_{\tau}^{C}(X)\left(t^{\prime}=\widehat{\sigma}[t]\right)\right\},
$$

is a subset of $W_{\tau}^{C}(X)$. Therefore, we consider $T_{\sigma}$ as a relation between $W_{\tau}^{C}(X)$ and $\widehat{\sigma}\left(W_{\tau}^{C}(X)\right)$, so that $T_{\sigma} \subseteq W_{\tau}^{C}(X) \times \widehat{\sigma}\left(W_{\tau}^{C}(X)\right)$. We notice that $T_{\sigma} \circ$ $\left(T_{\sigma}\right)^{-1}=T_{\sigma_{i d}}=\Delta_{W_{\tau}^{C}(X)}$ and $\left(T_{\sigma}\right)^{-1} \circ T_{\sigma}=\left\{\left(t, t^{\prime}\right) \mid \widehat{\sigma}[t]=\widehat{\sigma}\left[t^{\prime}\right]\right\}=\operatorname{ker} \sigma$ (the kernel of $\sigma)$. Then we have 
Proposition 10. Let $\sigma \in H y p_{R}^{C}(\tau)$ be a regular hypersubstitution of type $\tau$ and let $T_{\sigma}=W_{\tau}^{C}(X) \times \widehat{\sigma}\left(W_{\tau}^{C}(X)\right)$ be the corresponding tree transformation. Then $T_{\sigma}$ is bijective iff $\operatorname{ker} \sigma=\Delta_{W_{T}^{C}(X)}=T_{\sigma_{i d}}$.

Proof. $T_{\sigma}$ is bijective iff $T_{\sigma} \circ\left(T_{\sigma}\right)^{-1}=\left(T_{\sigma}\right)^{-1} \circ T_{\sigma}=T_{\sigma_{i d}}=\Delta_{W_{\tau}^{C}(X)}$. Now we use the previous remark.

\section{V-Transformations}

Definition 11. Let $V$ be a strong variety of partial algebras of type $\tau$ and $\sigma \in \operatorname{Hyp}_{R}^{C}(\tau)$. The set

$$
T_{\sigma}^{V}:=\left\{\left(t, t^{\prime}\right) \mid t, t^{\prime} \in W_{\tau}^{C}(X) \text { and } \widehat{\sigma}[t] \approx t^{\prime} \in I d^{s} V\right\}
$$

is called the $V$-transformation defined by the regular hypersubstitution $\sigma$.

Definition 12. ([2]) Let $V$ be a strong variety of partial algebras of type $\tau$. Two regular hypersubstitutions $\sigma_{1}, \sigma_{2} \in H y p_{R}^{C}(\tau)$ are called $V$-equivalent iff $\sigma_{1}\left(f_{i}\right) \approx \sigma_{2}\left(f_{i}\right) \in I d^{s} V$ for all $i \in I$. In this case we write $\sigma_{1} \sim_{V} \sigma_{2}$.

Theorem 13. ([2]) Let $V$ be a strong variety of partial algebras of type $\tau$, and let $\sigma_{1}, \sigma_{2} \in \operatorname{Hyp}_{R}^{C}(\tau)$. Then the following are equivalent:

(i) $\sigma_{1} \sim_{V} \sigma_{2}$.

(ii) For all $t \in W_{\tau}^{C}(X)$ the equation $\widehat{\sigma}_{1}[t] \approx \widehat{\sigma}_{2}[t] \in I d^{s} V$.

(iii) For all $\mathcal{A} \in V, \sigma_{1}(\mathcal{A})=\sigma_{2}(\mathcal{A})$.

Proposition 14. ([2]) Let $V$ be a strong variety of partial algebras of type $\tau$. If $\sigma_{1} \sim_{V} \sigma_{2}$ and $\widehat{\sigma}_{1}[s] \approx \widehat{\sigma}_{1}[t] \in I d^{s} V$, then $\widehat{\sigma}_{2}[s] \approx \widehat{\sigma}_{2}[t] \in I d^{s} V$ when $\sigma_{1}, \sigma_{2} \in \operatorname{Hyp}_{R}^{C}(\tau)$ and $s, t \in W_{\tau}^{C}(X)$.

Proposition 15. If $\sigma_{1} \sim_{V} \sigma_{2}$ then $T_{\sigma_{1}}^{V}=T_{\sigma_{2}}^{V}$.

Proof. We have only to show the inclusion $T_{\sigma_{1}}^{V} \subseteq T_{\sigma_{2}}^{V}$. Let $\sigma_{1} \sim_{V} \sigma_{2}$ and $\left(t, t^{\prime}\right) \in T_{\sigma_{1}}^{V}$. Then by Theorem 13(ii) we have $\widehat{\sigma}_{1}[t] \approx \widehat{\sigma}_{2}[t] \in I d^{s} V$ for all terms $t \in W_{\tau}^{C}(X)$ and $\widehat{\sigma}_{1}[t] \approx t^{\prime} \in I d^{s} V$. Therefore $\left(t, t^{\prime}\right) \in T_{\sigma_{2}}^{V}$ and $T_{\sigma_{1}}^{V} \subseteq T_{\sigma_{2}}^{V}$.

We are interested in all hypersubstitutions from $H y p_{R}^{C}(\tau)$ which preserve all strong identities from $V$. Such hypersubstitutions are called $V$-proper.

Definition 16. ([2]) Let $V$ be a strong variety of partial algebras of type $\tau$. A regular hypersubstitution $\sigma \in H y p_{R}^{C}(\tau)$ is called a $V$-proper hypersubstitution if for every $s \approx t \in I d^{s} V$, we have $\widehat{\sigma}[s] \approx \widehat{\sigma}[t] \in I d^{s} V$. 
We use $P(V)$ for the set of all V-proper hypersubstitutions of type $\tau$.

Proposition 17. ([2]) The algebra $\left(P(V) ; \circ_{h}, \sigma_{i d}\right)$ is a submonoid of $\left(\operatorname{Hyp}_{R}^{C}(\tau) ; o_{h}, \sigma_{i d}\right)$.

Now we compare the usual product of binary relations with the product of regular hypersubstitutions.

Proposition 18. If $V$ is a strong variety of partial algebras of type $\tau$, $\sigma_{1} \in P(V)$, and $\sigma_{2} \in W_{\tau}^{C}(X)$, then

$$
T_{\sigma_{1}}^{V} \circ T_{\sigma_{2}}^{V}=T_{\sigma_{1}{ }_{h} \sigma_{2}}^{V}
$$

Proof. Indeed, we have $\left(t, t^{\prime}\right) \in T_{\sigma_{1}}^{V} \circ T_{\sigma_{2}}^{V}$ iff there is a term $t^{\prime \prime}$ such that $\left(t, t^{\prime \prime}\right) \in T_{\sigma_{2}}^{V}$ and $\left(t^{\prime \prime}, t^{\prime}\right) \in T_{\sigma_{1}}^{V}$ iff $\widehat{\sigma}_{2}[t] \approx t^{\prime \prime} \in I d^{s} V$ and $\widehat{\sigma}_{1}\left[t^{\prime \prime}\right] \approx t^{\prime} \in I d^{s} V$. Since $\sigma_{1} \in P(V)$, we conclude that $\widehat{\sigma}_{1}\left[\widehat{\sigma}_{2}[t]\right] \approx \widehat{\sigma}_{1}\left[t^{\prime \prime}\right] \approx t^{\prime} \in I d^{s} V$ and therefore $\left(\sigma_{1} \circ_{h} \sigma_{2}\right)^{\wedge}[t] \approx t^{\prime} \in I d^{s} V$ and thus $\left(t, t^{\prime}\right) \in T_{\sigma_{1} \circ_{h} \sigma_{2}}^{V}$. This shows, $T_{\sigma_{1}}^{V} \circ T_{\sigma_{2}}^{V} \subseteq$ $T_{\sigma_{1} \circ_{h} \sigma_{2}}^{V}$

If conversely, $\left(t, t^{\prime}\right) \in T_{\sigma_{1} \circ_{h} \sigma_{2}}^{V}$ then $\left(\sigma_{1} \circ_{h} \sigma_{2}\right)^{\wedge}[t] \approx t^{\prime} \in I d^{s} V$ and then $\widehat{\sigma}_{1}\left[\widehat{\sigma}_{2}[t]\right] \approx t^{\prime} \in I d^{s} V$ with $t^{\prime \prime} \approx \widehat{\sigma}_{2}[t] \in I d^{s} V$ we have $\widehat{\sigma}_{1}\left[t^{\prime \prime}\right] \approx t^{\prime} \in I d^{s} V$ (because that $\left.\sigma_{1} \in P(V)\right)$ and then $\left(t, t^{\prime \prime}\right) \in T_{\sigma_{2}}^{V},\left(t^{\prime \prime}, t^{\prime}\right) \in T_{\sigma_{1}}^{V}$ and therefore $\left(t, t^{\prime}\right) \in T_{\sigma_{1}}^{V} \circ T_{\sigma_{2}}^{V}$. This shows the inclusion $T_{\sigma_{1}{ }_{h} \sigma_{2}}^{V} \subseteq T_{\sigma_{1}}^{V} \circ T_{\sigma_{2}}^{V}$ and altogether we have equality.

On the set $\mathcal{T}_{P(V)}:=\left\{T_{\sigma}^{V} \mid \sigma \in P(V)\right\}$ we may take the relational product as a binary relation. Indeed, if $\sigma_{1}, \sigma_{2} \in P(V)$ then by Proposition 17 from $T_{\sigma_{1}}^{V}, T_{\sigma_{2}}^{V} \in \mathcal{T}_{P(V)}$ we obtain $T_{\sigma_{1}}^{V} \circ T_{\sigma_{2}}^{V} \in \mathcal{T}_{P(V)}$. Since $\sigma_{i d} \in P(V)$, the $V$-transformation $T_{\sigma_{i d}}^{V}$ serves as identity element and we obtain a monoid $\left(\mathcal{T}_{P(V)} ; \circ, T_{\sigma_{i d}}^{V}\right)$.

Proposition 19. The monoid $\left(\mathcal{T}_{P(V)} ; \circ, T_{\sigma_{i d}}^{V}\right)$ is a homomorphic image of $\left(P(V) ; \circ_{h}, \sigma_{i d}\right)$.

Proof. A homomorphism $\varphi: P(V) \rightarrow \mathcal{T}_{P(V)}$ is defined by

$$
\varphi(\sigma):=T_{\sigma}^{V}
$$

Indeed, we have

$$
\varphi\left(\sigma_{1} \circ_{h} \sigma_{2}\right)=T_{\sigma_{1} \circ_{h} \sigma_{2}}^{V}=T_{\sigma_{1}}^{V} \circ T_{\sigma_{2}}^{V}=\varphi\left(\sigma_{1}\right) \circ \varphi\left(\sigma_{2}\right)
$$

and $\varphi\left(\sigma_{i d}\right)=T_{\sigma_{i d}}^{V}$.

Note that $\varphi$ is not one-to-one because of Proposition 15 and the kernel of $\varphi$ agrees with the relation $\sim_{V}$. 
Definition 20. ([2]) A regular hypersubstitution $\sigma \in H y p_{R}^{C}(\tau)$ is called an inner hypersubstitution of a strong variety $V$ of partial algebras of type $\tau$ if for every $i \in I$,

$$
\widehat{\sigma}\left[f_{i}\left(x_{1}, \ldots, x_{n_{i}}\right)\right] \approx f_{i}\left(x_{1}, \ldots, x_{n_{i}}\right) \in I d^{s} V .
$$

Let $P_{0}(V)$ be the set of all inner hypersubstitutions of $V$.

By definition $P_{0}(V)$ is the equivalence class $\left[\sigma_{i d}\right]_{\sim_{V}}$.

Note further $T_{\sigma_{i d}}^{V}$ is not the only identity element of $\left(\mathcal{T}_{P(V)} ; \circ, T_{\sigma_{i d}}^{V}\right)$ since all $T_{\sigma}^{V}$ with $\sigma \sim_{V} \sigma_{i d}$, i.e. where $\sigma$ are inner hypersubstitutions are equal to $T_{\sigma_{i d}}^{V}$ (Proposition 15).

Proposition 21. ([2]) The algebra $\left(P_{0}(V) ; \circ_{h}, \sigma_{i d}\right)$ is a submonoid of $\left(P(V) ; \circ_{h}, \sigma_{i d}\right)$.

Proposition 22. Let $V$ be a strong variety of partial algebras of type $\tau$ and let $\sigma \in H y p_{R}^{C}(\tau)$. Then $T_{\sigma}^{V}$ is reflexive iff $\sigma \in P_{0}(V)$.

Proof. If $T_{\sigma}^{V}$ is reflexive, then $\widehat{\sigma}[t] \approx t \in I d^{s} V$ for all $t \in W_{\tau}^{C}(X)$. This is valid also for $t=f_{i}\left(x_{1}, \ldots, t_{n_{i}}\right), i \in I$ and then $\widehat{\sigma}\left[f_{i}\left(x_{1}, \ldots, t_{n_{i}}\right)\right] \approx f_{i}\left(x_{1}, \ldots, t_{n_{i}}\right)$ $\in I d^{s} V$, i.e. $\widehat{\sigma}\left[f_{i}\left(x_{1}, \ldots, t_{n_{i}}\right)\right] \approx \widehat{\sigma}_{i d}\left[f_{i}\left(x_{1}, \ldots, t_{n_{i}}\right)\right] \in I d^{s} V$ and $\sigma \sim_{V} \sigma_{i d}$. Therefore $\sigma \in P_{0}(V)$. If conversely, $\sigma \in P_{0}(V)$ then $\sigma \sim_{V} \sigma_{i d}$ and by Proposition $21 \hat{\sigma}[t] \approx t \in I d^{s} V$ for $t \in W_{\tau}^{C}(X)$, but this means $(t, t) \in T_{\sigma}^{V}$ and $T_{\sigma}^{V}$ is reflexive.

The next, we introduce the concept of a semantical kernel.

Definition 23. Let $\sigma \in H y p_{R}^{C}(\tau)$ and let $V$ be a strong variety of partial algebras of type $\tau$. The set

$$
K e r_{V} \sigma:=\left\{\left(t, t^{\prime}\right) \mid t, t^{\prime} \in W_{\tau}^{C}(X) \text { and } \widehat{\sigma}[t] \approx \widehat{\sigma}\left[t^{\prime}\right] \in I d^{s} V\right\}
$$

will be called the kernel of $\sigma$ with respect to $V$ or the semantical kernel of $\sigma$.

Proposition 24. If $\sigma_{1} \sim_{V} \sigma_{2}$ then $\operatorname{Ker}_{V} \sigma_{1}=K e r_{V} \sigma_{2}$.

Proof. By Proposition 14.

Proposition 25. Let $V$ be a strong variety of partial algebras of type $\tau$ and let $\sigma \in \operatorname{Hyp}_{R}^{C}(\tau)$. Then

$$
\operatorname{ker}_{V} \sigma \subseteq \operatorname{ker}_{V}\left(\rho \circ_{h} \sigma\right)
$$

for all $\rho \in P(V)$. 
Proof. For any $\left(t, t^{\prime}\right) \in \operatorname{ker}_{V} \sigma$ we have $\widehat{\sigma}[t] \approx \widehat{\sigma}\left[t^{\prime}\right] \in I d^{s} V$. Since $\rho$ is a $V$-proper hypersubstitution, this implies that $\widehat{\rho}[\widehat{\sigma}[t]] \approx \widehat{\rho}\left[\widehat{\sigma}\left[t^{\prime}\right]\right] \in I d^{s} V$, and so $\left(t, t^{\prime}\right) \in \operatorname{ker}_{V}\left(\rho \circ_{h} \sigma\right)$.

Proposition 26. Let $V$ be a strong variety of partial algebras of type $\tau$ and let $\sigma \in \operatorname{Hyp}_{R}^{C}(\tau)$. Then $\left(T_{\sigma}^{V}\right)^{-1} \circ T_{\sigma}^{V}=K e r_{V} \sigma$.

$$
\begin{aligned}
\text { Proof. We have } & \\
\left(t, t^{\prime \prime}\right) \in\left(T_{\sigma}^{V}\right)^{-1} \circ T_{\sigma}^{V} & \Leftrightarrow \exists t^{\prime}\left(\left(t, t^{\prime}\right) \in T_{\sigma}^{V} \text { and }\left(t^{\prime}, t^{\prime \prime}\right) \in\left(T_{\sigma}^{V}\right)^{-1}\right) \\
& \Leftrightarrow \exists t^{\prime}\left(\left(t, t^{\prime}\right) \in T_{\sigma}^{V} \text { and }\left(t^{\prime \prime}, t^{\prime}\right) \in T_{\sigma}^{V}\right) \\
& \Leftrightarrow \exists t^{\prime}\left(\widehat{\sigma}[t] \approx t^{\prime} \in I d^{s} V \text { and } \widehat{\sigma}\left[t^{\prime \prime}\right] \approx t^{\prime} \in I d^{s} V\right) \\
& \Leftrightarrow \widehat{\sigma}[t] \approx \widehat{\sigma}\left[t^{\prime \prime}\right] \in I d^{s} V \\
& \Leftrightarrow\left(t, t^{\prime \prime}\right) \in \operatorname{ker}_{V} \sigma .
\end{aligned}
$$

Proposition 27. For a $V$-proper hypersubstitution $\sigma$ the following are equivalent:

(i) $T_{\sigma}^{V}$ is transitive.

(ii) $\sigma \circ_{h} \sigma \sim_{V} \sigma$.

(iii) $T_{\sigma}^{V} \subseteq k e r_{V} \sigma$.

Proof. (i) $\Rightarrow$ (ii): If $T_{\sigma}^{V}$ is transitive, then $T_{\sigma}^{V} \circ T_{\sigma}^{V} \subseteq T_{\sigma}^{V}$ and therefore $T_{\sigma}^{V} \circ T_{\sigma}^{V}=T_{\sigma \circ_{h} \sigma}^{V} \subseteq T_{\sigma}^{V}$. This means, if $\left(t, t^{\prime}\right) \in T_{\sigma \circ_{h} \sigma}^{V}$, i.e. $\left(\sigma \circ_{h} \sigma\right)^{\wedge}[t] \approx t^{\prime} \in I d^{s} V$ then $\left(t, t^{\prime}\right) \in T_{\sigma}^{V}$ i.e. $\widehat{\sigma}[t] \approx t^{\prime} \in I d^{s} V$. But $\left(\sigma \circ_{h} \sigma\right)^{\wedge}[t] \approx \widehat{\sigma}[t] \in I d^{s} V$ for all $t \in W_{\tau}^{C}(X)$ and there $\sigma \circ_{h} \sigma \sim_{V} \sigma$ by Theorem 13 .

(ii) $\Rightarrow\left(\right.$ i): If $\sigma \circ_{h} \sigma \sim_{V} \sigma$ then by Proposition $15 T_{\sigma \circ_{h} \sigma}^{V}=T_{\sigma}^{V}$ and Proposition $18, T_{\sigma}^{V} \circ T_{\sigma}^{V}=T_{\sigma \circ_{h} \sigma}^{V}=T_{\sigma}^{V}$ and $T_{\sigma}^{V}$ is transitive.

(ii) $\Rightarrow$ (iii): Assume that $\sigma \circ_{h} \sigma \sim_{V} \sigma$ and that $\left(t, t^{\prime}\right) \in T_{\sigma}^{V}$, i.e. $\widehat{\sigma}[t] \approx$ $t^{\prime} \in I d^{s} V$. Then $\widehat{\sigma}[\widehat{\sigma}[t]] \approx \widehat{\sigma}\left[t^{\prime}\right] \in I d^{s} V$ since $\sigma$ is $V$-proper and we have $\left(\sigma \circ_{h} \sigma\right)^{\wedge}[t] \approx \widehat{\sigma}\left[t^{\prime}\right] \in I d^{s} V$. From $\left(\sigma \circ_{h} \sigma\right)^{\wedge}[t] \approx \widehat{\sigma}\left[t^{\prime}\right] \in I d^{s} V$ we obtain $\widehat{\sigma}[t] \approx \widehat{\sigma}\left[t^{\prime}\right] \in I d^{s} V$ and then $\left(t, t^{\prime}\right) \in k e r_{V} \sigma$. This show $T_{\sigma}^{V} \subseteq k e r_{V} \sigma$.

(iii) $\Rightarrow$ (ii): If $T_{\sigma}^{V} \subseteq \operatorname{ker}_{V} \sigma$ then $\left(t, t^{\prime}\right) \in T_{\sigma}^{V}$, i.e. $\widehat{\sigma}[t] \approx t^{\prime} \in I d^{s} V$ and since $\sigma$ is $V$-proper we get $\widehat{\sigma}[\widehat{\sigma}[t]] \approx \widehat{\sigma}\left[t^{\prime}\right] \in I d^{s} V$. Since $\left(t, t^{\prime}\right) \in \operatorname{ker}_{V} \sigma$ we have $\widehat{\sigma}[t] \approx \widehat{\sigma}\left[t^{\prime}\right] \in I d^{s} V$ and then $\widehat{\sigma}[\widehat{\sigma}[t]] \approx \widehat{\sigma}[t] \in I d^{s} V$, i.e. $\sigma \circ_{h} \sigma \sim_{V} \sigma$.

Proposition 28. Let $V$ be a strong variety of partial algebras of type $\tau$. Then:

(i) $\quad T_{\sigma}^{V}$ is surjective iff $T_{\sigma}^{V} \circ\left(T_{\sigma}^{V}\right)^{-1}=I d^{s} V$.

(ii) $T_{\sigma}^{V}$ is injective iff $\left(T_{\sigma}^{V}\right)^{-1} \circ T_{\sigma}^{V}=k e r_{V} \sigma=\triangle_{W_{T}^{C}(X)}$.

(iii) $T_{\sigma}^{V}$ is bijective iff $T_{\sigma}^{V} \circ\left(T_{\sigma}^{V}\right)^{-1}=\left(T_{\sigma}^{V}\right)^{-1} \circ T_{\sigma}^{V}=\triangle_{W_{\tau}^{C}(X)}$. 
Proof. (i) Assume that $T_{\sigma}^{V}$ is surjective. To show the equality $T_{\sigma}^{V} \circ\left(T_{\sigma}^{V}\right)^{-1}=$ $I d^{s} V$. Assume that $t \approx t^{\prime} \in I d^{s} V$. Since $T_{\sigma}^{V}$ is surjective, to $t^{\prime}$ there is a term $t^{\prime \prime}$ such that $\left(t^{\prime \prime}, t^{\prime}\right) \in T_{\sigma}^{V}$, i.e. such that $\widehat{\sigma}\left[t^{\prime \prime}\right] \approx t^{\prime} \in I d^{s} V$. Then we have also $\widehat{\sigma}\left[t^{\prime \prime}\right] \approx t \in I d^{s} V$ and $\left(t^{\prime \prime}, t\right) \in T_{\sigma}^{V}$, i.e. $\left(t, t^{\prime \prime}\right) \in\left(T_{\sigma}^{V}\right)^{-1}$. Altogether, this gives $\left(t, t^{\prime}\right) \in T_{\sigma}^{V} \circ\left(T_{\sigma}^{V}\right)^{-1}$ and thus $I d^{s} V \subseteq T_{\sigma}^{V} \circ\left(T_{\sigma}^{V}\right)^{-1}$. The next, we assume that $\left(t, t^{\prime}\right) \in T_{\sigma}^{V} \circ\left(T_{\sigma}^{V}\right)^{-1}$ then there exist $t^{\prime \prime}$ such that $\left(t, t^{\prime \prime}\right) \in\left(T_{\sigma}^{V}\right)^{-1}$ and $\left(t^{\prime \prime}, t^{\prime}\right) \in T_{\sigma}^{V}$. We have $\left(t^{\prime \prime}, t\right) \in T_{\sigma}^{V}$ and $\left(t^{\prime \prime}, t^{\prime}\right) \in T_{\sigma}^{V}$ i.e. $\widehat{\sigma}\left[t^{\prime \prime}\right] \approx t \in I d^{s} V$ and $\widehat{\sigma}\left[t^{\prime \prime}\right] \approx t^{\prime} \in I d^{s} V$. So, $t \approx t^{\prime} \in I d^{s} V$. Conversely, we assume that $T_{\sigma}^{V} \circ\left(T_{\sigma}^{V}\right)^{-1}=I d^{s} V$. Let $t \in W_{\tau}^{C}(X)$ be an arbitrary term of $W_{\tau}^{C}(X)$. We have to show that there is a term $t^{\prime} \in W_{\tau}^{C}(X)$ with $\widehat{\sigma}\left[t^{\prime}\right] \approx t \in I d^{s} V$. From $t \approx t \in I d^{s} V=T_{\sigma}^{V} \circ\left(T_{\sigma}^{V}\right)^{-1}$ we obtain the existence of a term $t^{\prime} \in W_{\tau}^{C}(X)$ such that $\left(t^{\prime}, t\right) \in T_{\sigma}^{V}$, but this means $\widehat{\sigma}\left[t^{\prime}\right] \approx t \in I d^{s} V$ and this shows surjectivity.

(ii) $\left(T_{\sigma}^{V}\right)^{-1} \circ T_{\sigma}^{V}=\operatorname{Ker}_{V} \sigma$ is clear. Assume that $T_{\sigma}^{V}$ is injective and let $\left(t, t^{\prime}\right) \in\left(T_{\sigma}^{V}\right)^{-1} \circ T_{\sigma}^{V}$. Then we have $\left(t, t^{\prime \prime}\right) \in T_{\sigma}^{V}$ and $\left(t^{\prime}, t^{\prime \prime}\right) \in T_{\sigma}^{V}$ there follows $t=t^{\prime}$. We get $\left(t, t^{\prime}\right) \in \triangle_{W_{\tau}^{C}(X)}$ and thus $\left(T_{\sigma}^{V}\right)^{-1} \circ T_{\sigma}^{V} \subseteq \triangle_{W_{\tau}^{C}(X)}$. If conversely $\left(t, t^{\prime}\right) \in \triangle_{W_{\tau}^{C}(X)}$ then $t=t^{\prime}$ and $\widehat{\sigma}[t]=\widehat{\sigma}\left[t^{\prime}\right]=: t^{\prime \prime}$. Then we have also $\widehat{\sigma}[t] \approx t^{\prime \prime} \in I d^{s} V$ and $\widehat{\sigma}\left[t^{\prime}\right] \approx t^{\prime \prime} \in I d^{s} V$ and $\left(t, t^{\prime \prime}\right) \in T_{\sigma}^{V},\left(t^{\prime}, t^{\prime \prime}\right) \in T_{\sigma}^{V}$ and then $\left(t, t^{\prime}\right) \in\left(T_{\sigma}^{V}\right)^{-1} \circ T_{\sigma}^{V}$, i.e. $\triangle_{W_{\tau}^{C}(X)} \subseteq\left(T_{\sigma}^{V}\right)^{-1} \circ T_{\sigma}^{V}$. Altogether, this gives $\left(T_{\sigma}^{V}\right)^{-1} \circ T_{\sigma}^{V}=\triangle_{W_{\tau}^{C}(X)}$. Assume now that $\left(T_{\sigma}^{V}\right)^{-1} \circ T_{\sigma}^{V}=\triangle_{W_{\tau}^{C}(X)}$ and that $\left(t, t^{\prime \prime}\right) \in T_{\sigma}^{V}$ and $\left(t^{\prime}, t^{\prime \prime}\right) \in T_{\sigma}^{V}$. Then $\left(t, t^{\prime}\right) \in\left(T_{\sigma}^{V}\right)^{-1} \circ T_{\sigma}^{V}=\triangle_{W_{\tau}^{C}(X)}$, i.e. $t=t^{\prime}$ and therefore $T_{\sigma}^{V}$ is injective.

(iii) Assume that $T_{\sigma}^{V}$ is bijective. Since $\sigma$ is $V$-proper, we have by (i) and (ii):

$$
T_{\sigma}^{V} \circ\left(T_{\sigma}^{V}\right)^{-1}=I d^{s} V \subseteq \operatorname{ker}_{V} \sigma=\left(T_{\sigma}^{V}\right)^{-1} \circ T_{\sigma}^{V}=\triangle_{W_{\tau}^{C}(X)}
$$

and therefore $I d^{s} V=\triangle_{W_{\tau}^{C}(X)}$ and $T_{\sigma}^{V} \circ\left(T_{\sigma}^{V}\right)^{-1}=\left(T_{\sigma}^{V}\right)^{-1} \circ T_{\sigma}^{V}=\triangle_{W_{\tau}^{C}(X)}$.

If conversely, $T_{\sigma}^{V} \circ\left(T_{\sigma}^{V}\right)^{-1}=\left(T_{\sigma}^{V}\right)^{-1} \circ T_{\sigma}^{V}=\triangle_{W_{\tau}^{C}(X)}$, then by (ii) $T_{\sigma}^{V}$ is injective. Therfore, $T_{\sigma}^{V}=\left\{\left(t, t^{\prime}\right) \mid \widehat{\sigma}[t] \approx t^{\prime} \in I d^{s} V\right\}=I d^{s} V=\triangle_{W_{\tau}^{C}(X)}$.

\section{References}

[1] F. Börner, Varieties of Partial Algebras, Beiträge zur Algebra und Geometrie, Vol. 37 (1996), No. 2, 259-287.

[2] S. Busaman, K. Denecke, Unsolid and Fluid Strong Varieties of Partial Algebras, preprint 2006, submitted to Int. Journal of Mathematics and Mathematical Sciences.

[3] J. Płonka, On hyperidentities of some varieties, General Algebra and Discrete Mathematics, Heldermann-Verlag, Berlin (1995), 199-214. 
[4] B. Staruch, B. Staruch, Strong regular varieties of partial algebras, Algebra Universalis, 31 (1994), 157-176.

[5] D. Welke, Hyperidentitäten partieller Algebren, Ph.D.Thesis, Universität Potsdam, Potsdam (1996). 